\title{
Immunofluorescence in Oral Pathology: Part I-Methodology
}

\author{
${ }^{1}$ Premalatha BR, ${ }^{2}$ Roopa S Rao, ${ }^{3}$ Vijaya Mysorekar \\ ${ }^{1}$ Senior Lecturer, Department of Oral Pathology, MS Ramaiah Dental College and Hospital, Bengaluru, Karnataka, India \\ ${ }^{2}$ Professor, Department of Oral Pathology, MS Ramaiah Dental College and Hospital, Bengaluru, Karnataka, India \\ ${ }^{3}$ Professor, Department of Pathology, MS Ramaiah Medical College and Hospital, Bengaluru, Karnataka, India
}

Correspondence: Roopa S Rao, Professor, Department of Oral Pathology, MS Ramaiah Dental College and Hospital, Bengaluru Karnataka, India, e-mail: drroopasrao1971@gmail.com

\section{ABSTRACT}

Immunofluorescence is an immunological method used to demonstrate the presence of antigen and antibodies in tissues or serum. Immunofluorescence techniques have become indispensable in modern pathology laboratories and have greatly contributed to the diagnosis, treatment and understanding of autoimmune bullous diseases, hereditary bullous diseases, collagen-vascular diseases, many inflammatory dermatosis and cutaneous tumors. This review emphasizes the basics of immunofluorescence techniques, methodology, interpretation of the results and the newer advancements in the field.

The application of immunofluorescence techniques in various vesiculobullous disorders will be extensively discussed in the subsequent parts. Part II will deal with the pemphigus group and other dermatological conditions with oral involvement and part III with subepidermal immunobullous diseases.

Keywords: Immunofluorescence, Immunobullous disorders, Direct immunofluorescence technique, Indirect immunofluorescence technique.

\section{INTRODUCTION}

Immunofluorescence (IF) is a histochemical laboratory staining technique used for demonstrating the presence of antibodies bound to antigens in tissues or serum. These techniques supplement clinical findings and histopathology in the diagnosis of immunobullous disorders. They permit early diagnosis, treatment and subsequent monitoring of these potentially life-threatening disorders. ${ }^{1}$ In addition, the IF methods are used in research to advance the understanding and classification of the immunobullous disorders. ${ }^{2}$

\section{APPLICATION}

The most important application is in the field of autoimmune diseases. It has wide application in demonstration of enzymes, hormones, plasma proteins, cells and its constituents and also in identifying viral, protozoal, bacterial and parasitic antigens. IF methods also have the potential to define antigen-antibody interactions at the subcellular level, such as the detection of antibodies against mitochondria, microsomes and smooth muscle fibers as well as identifying small cell surface structures, such as receptors on lymphocytes. ${ }^{3,4}$

\section{PRINCIPLE}

In IF techniques, antigens, antibodies or their complexes are viewed under a fluorescence microscope, using the corresponding antibodies complexed to a fluorochrome. ${ }^{5}$ The combination of antibody with its specific antigen does not lead to a visible change and therefore a readily identifiable label (fluorochrome) must be irreversibly bound to the antibody so that its localization can be recognized. ${ }^{4}$

Fluorescent materials give off light because of their atomic structure. Electrons are arranged in discrete energy levels. When an electron absorbs the energy from a photon of light it becomes 'excited' and jumps to a higher, less stable energy level. The excited state has a half-life of less than 10 seconds. The electron loses a small amount of energy as heat and the remainder is given off in the form of a photon. The emitted light has a lower energy than the absorbed light, hence has a longer wavelength. ${ }^{5,6}$ The emitted light is almost invariably in the visible spectrum ${ }^{4}$ (Fig. 1).

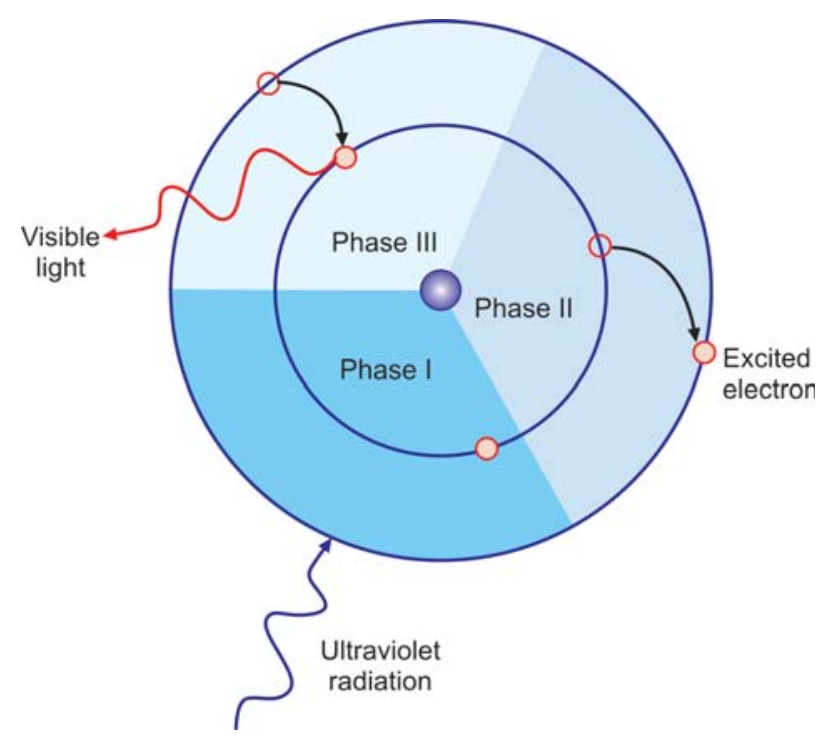

Fig. 1: Principle of fluorescence 
These fluorochrome markers are detected with a fluorescence microscope equipped with a mercury-vapor or xenon light source, and appropriate exciter and barrier filters. The exciter filter serves to shed light of necessary wavelength on the examined slide, while the barrier filter stops the exciting photons, letting through only the fluorescent light. ${ }^{5}$

\section{Collection and Handling of Specimens for Immunofluorescent Studies}

Two types of specimens can be examined by IF techniques, notably biopsy specimens of skin or mucous membranes and serum samples. ${ }^{7}$

\section{Biopsy Specimens}

Direct immunofluorescence (DIF) studies require a biopsy of the patient's skin or mucosa. ${ }^{2}$ Monkey or guinea pig esophagus, rat bladder epithelium or blister fluid can be used for indirect immunofluorescence (IIF). ${ }^{1,8}$

In preparation of the tissues prior to biopsy, topical steroids must be stopped at least 1 month before to prevent false-negative results. ${ }^{9} \mathrm{~A} 3$ to $4 \mathrm{~mm}$ punch biopsy is generally adequate. The choice of biopsy site is very important (Table 1$){ }^{2}$ In the autoimmune blistering disease group, an inflamed but unblistered perilesional area from the end of an elliptical biopsy and a $3 \mathrm{~mm}$ punch biopsy of uninvolved skin are adequate specimens. ${ }^{10}$ In some blistering disorders one of these alone will be sufficient. Biopsies of the lesions themselves are not satisfactory as immunoreactants and tissue structures may be altered, making interpretation difficult. ${ }^{2}$

Susana Mariela Sano et al (2008) studied 125 cases to assess the sensitivity of DIF in oral diseases and concluded that there are some anatomic oral regions in which the DIF technique sensitivity is greater: mouth floor, hard palate, superior labial mucosa and ventral side of the tongue. Knowing this may allow the physician to choose accessible regions, even those that are not close to the clinical lesions. They also concluded that punch biopsy samples have a greater detection rate for DIF than those taken with a scalpel. ${ }^{11}$

In dermatitis herpetiformis, due to often focal and skipping nature of the immunoreactants a shave biopsy provides broader surface to evaluate than a punch biopsy. ${ }^{10}$ In desquamative gingivitis secondary to mucous membrane pemphigoid, samples

Table 1: Biopsy site for $\mathrm{DIF}^{2}$

\begin{tabular}{ll}
\multicolumn{1}{c}{ Condition } & \multicolumn{1}{c}{ Biopsy site(s) } \\
$\begin{array}{l}\text { Pemphigus (all types) } \\
\text { Pemphigoid (all types) }\end{array}$ & $\begin{array}{l}\text { Perilesional and uninvolved (buttocks) } \\
\text { Perilesional and uninvolved } \\
\text { (thigh or arm) }\end{array}$ \\
$\begin{array}{l}\text { Linear IgA disease } \\
\text { Derilesional and uninvolved (buttocks) } \\
\text { Epidermolysis } \\
\text { bullosa aquisita }\end{array}$ & $\begin{array}{l}\text { Uninvolved } \\
\text { Pullous systemic } \\
\text { lupus erythematosis }\end{array}$ \\
\hline
\end{tabular}

can be obtained by 'peeling technique' where rubbing the perilesional affected gingivae with a cotton swab induces a fresh peeling of the mucosa. ${ }^{12}$ In autoimmune and inflammatory disorders other than autoimmune blistering diseases, (e.g. cutaneous lupus erythematosis, lichen planus, amyloidosis, vasculitis, porphyria), the specimen should be taken from the lesional areas. ${ }^{10,13}$ Biopsy from hyperkeratotic component (e.g. lichen planus, lupus erythematosis) should include that along with its adjacent erythematous mucosa. ${ }^{9}$

\section{Serum Samples}

Used in IIF techniques to detect circulating autoantibodies. Approximately $10 \mathrm{ml}$ of blood is collected in a tube without anticoagulant and transported to the laboratory within 48 hours. Serum is then centrifuged and stored at $-25^{\circ} \mathrm{C}$ until analysis is performed. Alternatively, separated serum may be sent to the laboratory within 1 month of collection. ${ }^{1,2,10,13}$

\section{Transportation of Biopsy Specimen}

Ideally, tissues are collected and snap-frozen immediately. In most cases, DIF facilities are not available and tissue specimens need to be transported to the laboratory. In such situations, biopsies should be washed in distilled water or normal saline and placed in Michel's transportation media. Washing removes blood proteins and maintains neutral $\mathrm{pH}$ in the medium. The media ensures immunoreactant preservation at ambient temperatures for up to 6 months. This facilitates mailing of specimens to the laboratory from a distance. In the laboratory, tissue specimens received in Michel's medium are washed in phosphate buffered saline (PBS) to remove ammonium salts and any residual blood proteins. The tissue is then snap-frozen. ${ }^{2}$

\section{Processing of Biopsy Specimen}

Quick freezing is the most widely used method for handling biopsy specimens for IF studies. It is performed by immersing the biopsy specimen either in liquid nitrogen or cold solid carbon dioxide or in a hexane bath. The quick frozen biopsy is then mounted in optimal cutting temperature (OCT) embedding compound and sectioned in a cryostat. Tissue substrates for IIF techniques are processed similarly. ${ }^{1}$

Fresh frozen tissue is preferred for this technique however, frequently, the material received by the laboratory has been fixed in formalin. Norman Firth et al have suggested that enzyme digestion of tissue sections glued to glass slides prior to incubation with antibodies gives comparable results with DIF on fresh frozen tissue. ${ }^{14}$

\section{IMMUNOFLUORESCENCE TECHNIQUES AND PROCEDURES}

In clinical immunodermatology, there are three basic types of immunofluorescence techniques: Direct, indirect and complement techniques. ${ }^{1}$

\section{Direct Immunofluorescence Technique (DIF)}

DIF is a one-step procedure used to detect and localize immunoreactants deposited in vivo in the patient's skin or mucosa $^{1,2}$ (Fig. 2). DIF must be considered when diagnosing 


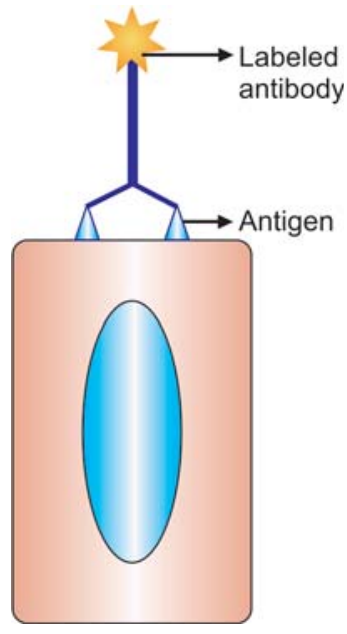

Fig. 2: Direct immunofluorescence technique

oral mucosal conditions characterized by chronic, multifocal or diffuse blisters, erosions, ulcers or combination of these patterns. $^{9}$

Ranjana Walker Minz et al (2010) analyzed the contribution of immunofluorescence in diagnosing bullous and nonbullous lesions of the skin in comparison with histopathology and clinical diagnosis and concluded that the DIF of skin in conjunction with histopathology gives the best diagnostic yield. It is invaluable in confirming the diagnosis of small vessel vasculitides and bullous lesions of skin and can be used as an additional tool to pinpoint the diagnosis of systemic and localized autoimmune diseases involving the skin. ${ }^{15}$

\section{Procedures (Fig. 3)}

Skin biopsy specimen: Biopsy may be taken with the help of a 3 to $4 \mathrm{~mm}$ punch from perilesional skin or mucosa. The specimen is either quick frozen or placed in transport medium. After removal from the media, the specimen is washed three times (10 minutes each) in PBS and then subsequently frozen. ${ }^{5}$

Quick freezing: The biopsy is placed in a labeled plastic container half filled with cryostat embedding OCT compound. The container is cooled either rapidly in liquid nitrogen or slowly in cryostat $\left(-20^{\circ} \mathrm{C}\right)$ until the edges are frozen and central parts remain fluid. The specimen is then oriented in the fluid part, covered with more OCT compound and frozen until the consistency of the block is appropriate for cryosectioning. ${ }^{16}$

Sectioning: The metal chunk bearing the frozen tissue specimen is placed in the cryostat. A 4 to $6 \mu \mathrm{m}$ thickness sections are cut and taken off the cryostat by gently touching with a glass slide. Tissue sections are separated from each other by straight lines by wax pencil, to prevent intermixing of fluorescent antisera. Alternatively, slides with special chambers to prevent intermixing can be used. The slides are then allowed to air dry for 15 minutes. $^{16}$

Incubation with fluorescein isothiocyanate (FITC) conjugates: After PBS rinsing, slides are overlaid in a moist chamber with FITC conjugate for 20 to 30 minutes at $37^{\circ} \mathrm{C} .{ }^{5}$ As a routine, the following antisera are recommended: anti-IgG, anti-IgA, antiIgM, anti-C3 and antifibrinogen. Other antisera are used in
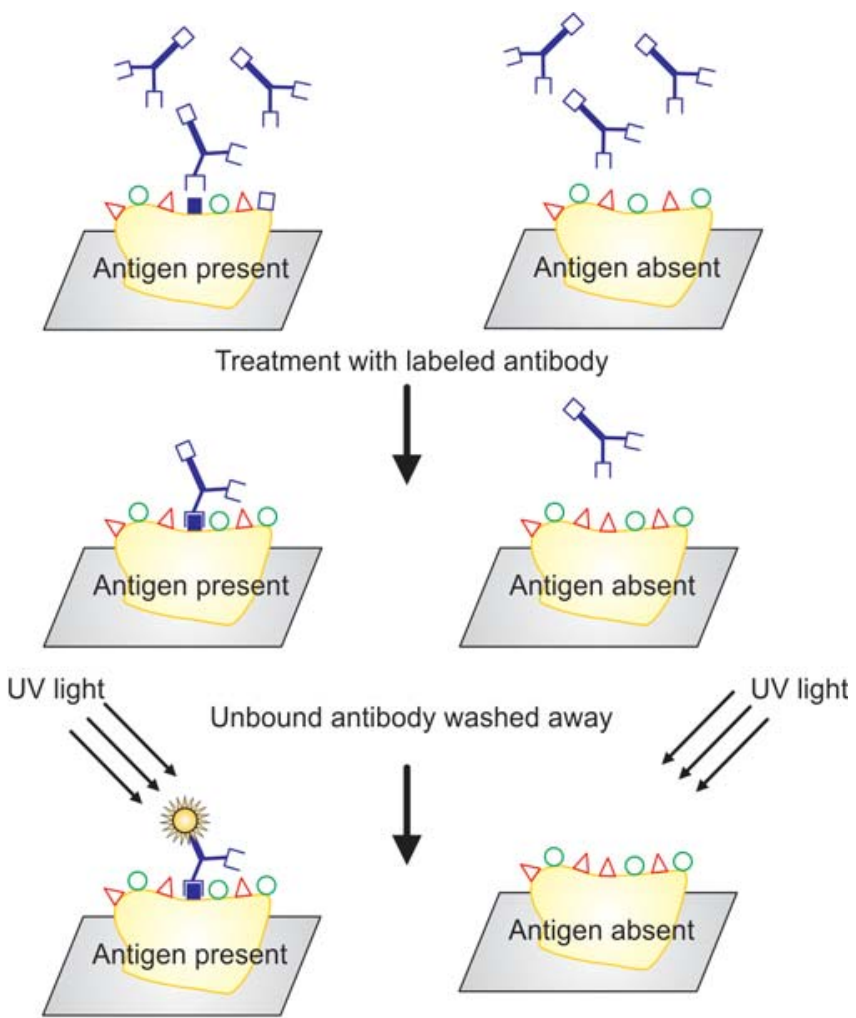

Fluorescence observed, where antigen is located

Fig. 3: Direct immunofluorescence procedure

special circumstances. The unreacted antiserum is washed off in three (10 minutes) changes of PBS. The slides are then drained and the excess buffer is wiped with dry cotton gauze. Then the slides are mounted in buffered glycerin and examined under fluorescence microscope. ${ }^{16}$

Controls: In staining with labeled antibodies, as in any serologic method, positive and negative controls constitute an integral part.

Interpretation: It is based on a combination of both the positive and negative observations from each of the five conjugated antisera. Microscopic evaluation includes both the location and the pattern of fluorescence seen with each reagent. ${ }^{9}$ Immunoreactants are deposited in two main patterns: In the epidermal intercellular space (ICS) either as diffuse or focal deposition and along the basement membrane zone (BMZ) as smooth and linear, granular and discontinuous deposits or a combination of the two. ${ }^{2}$

\section{Indirect Immunofluorescence Technique (IIF)}

IIF is a two-step serological technique used to identify circulating autoantibodies in a patient's serum. These antibodies are most commonly of $\lg$ or $\lg A$ classes $^{1,2}$ (Fig. 4). It is 10 times more sensitive than DIF. ${ }^{5}$ However, indirect technique is less applicable in the diagnosis of oral manifestations of mucocutaneous diseases. ${ }^{9}$

\section{Procedures}

Serum sample: Patient's blood (5-10 ml) is taken, centrifuged to extract serum and then serial dilutions $(1: 10,1: 20,1: 40)$ are made. $^{5}$ 


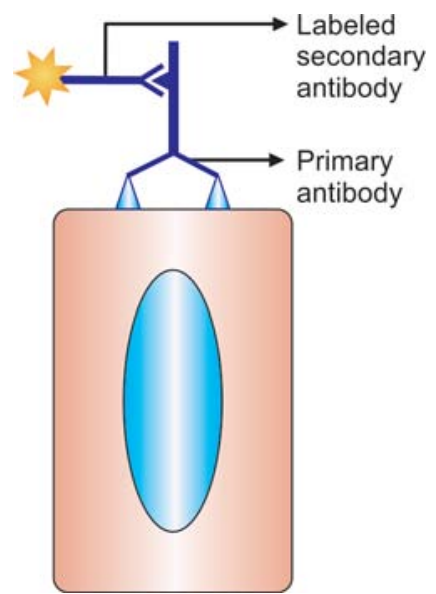

Fig. 4: Indirect immunofluorescence technique

First step: This consists of contact between the serum and sections of appropriate substrate, which contains the corresponding antigen. ${ }^{5}$ The initial serum dilution to $1: 10$ or $1: 80$ is incubated with the frozen sections of the substrate for 30 minutes at $37^{\circ} \mathrm{C}$. If positive, subsequent incubations are used with increasingly higher dilutions of sera. Autoantibodies in the serum bind to components of the epidermis and BMZ. Unbound serum is removed by three (10 minutes) washes in PBS. ${ }^{2}$

Second step: This step is identical to the staining procedure of the DIF technique. Sections are treated with FITC conjugated anti-IgG, IgA, IgM at $37^{\circ} \mathrm{C}$ for 30 minutes. ${ }^{5}$ Positive serum and negative serum are incubated in similar fashion. ${ }^{2}$ After the final three washes in PBS, the sections are air-dried, mounted with buffered glycerol and examined with a fluorescence microscope. ${ }^{5}$

Controls: Every series of IIF studies must include known positive and negative controls. For the negative controls, sections are incubated with normal human serum and with FITC conjugated antihuman lgG. ${ }^{2}$

Interpretation: The class or subclass of immunoglobulins, the pattern and the site of deposition are noted. ${ }^{2}$

Epithelial substrate: A large number of tissue substrates are used for IIF including monkey esophagus, guinea pig lip/ esophagus, rabbit lip/esophagus, rodent urinary bladder and normal human skin. ${ }^{5}$

\section{Complement Binding Indirect Immunofluorescence Technique}

This is a very sensitive, three-step serologic technique, by which very small quantities of circulating antibodies are detected by means of their high affinity to fix complement. ${ }^{1,5}$ The compliment can be demonstrated using FITC labeled anticomplement. ${ }^{3}$ The technique is more sensitive than routine IIF due to the amplification achieved through binding of more than one molecule of complement to each immunoglobulin and the subsequent visualization of the multiple molecules of complement $^{2}$ (Fig. 5).

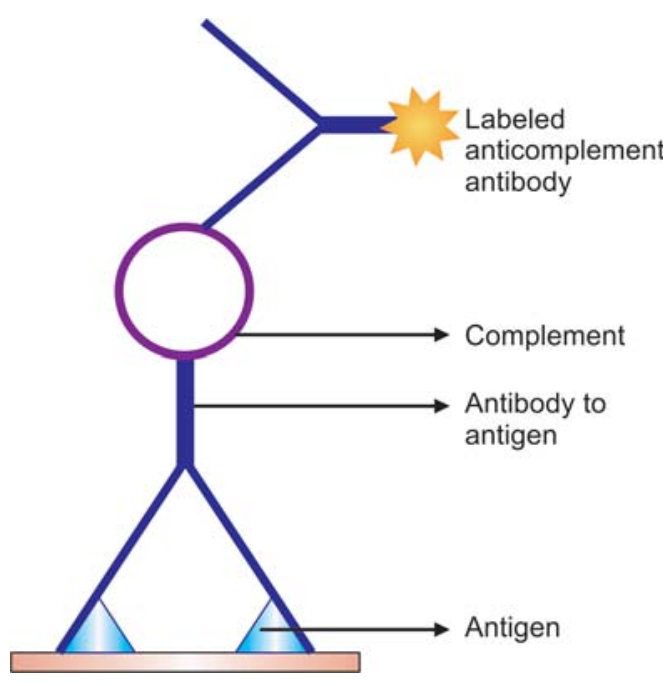

Fig. 5: Complement fixation technique

\section{Procedures}

First step: This step is analogous to the first step of IIF, except that the complement in the serum is inactivated before hand by heating for 30 minutes at $56^{\circ} \mathrm{C}$. If present, serum complementfixing antibodies bind to the antigen, during incubation with the normal tissue substrate. ${ }^{5}$ Specimens are then washed. ${ }^{1}$

Second step: The tissue sections are overlaid with a source of complement, usually a fresh normal human or guinea pig serum. The complement-fixing IgG/IgM antibodies already bound to the antigen activate the complement cascade and generate numerous molecules of C3 at the same time. ${ }^{5}$ Specimens are then washed. ${ }^{1}$

Third step: It consists of revealing complement C3 by staining the sections with FITC conjugate antihuman C3 antibody. Sections are washed in PBS after different incubations and examined under a fluorescence microscope. As in the indirect technique, positive and negative control sera are applied.,17

\section{VARIANTS OF IMMUNOFLUORESCENCE TECHNIQUES}

\section{Salt-Split Technique}

This technique is used to distinguish between subepidermal blistering conditions with similar DIF findings like bullous pemphigoid (BP) and epidermolysis bullosa aquisita (EBA). ${ }^{1}$ Various methods exist to split skin through the lamina lucida, this being the area of least resistance in the BMZ. These methods include suction blister creation or incubation in sodium chloride, trypsin and PBS. Cleavage through the lamina lucida places the hemidesmosomes and upper lamina lucida in the roof of the split and the lower lamina lucida and the sublamina densa in the floor. ${ }^{2}$

\section{Procedures}

This technique may be used with biopsies of the patient's skin for DIF or skin substrate for IIF. ${ }^{2}$ 
DIF on salt-split specimen: Perilesional skin of the patient is processed in $1 \mathrm{M} \mathrm{NaCl}$ solution for 24 hours in order to induce a dermal-epidermal separation at the level of lamina lucida and then subjected to the standard DIF procedure. ${ }^{5}$

IIF on salt-split substrate: The selected substrate is incubated with $1 \mathrm{M} \mathrm{NaCl}$ solution for 72 hours to create an artificial cleavage at the level of lamina lucida and then processed as for routine IIF. ${ }^{5}$

Interpretation: $\mathrm{BP}$ antibodies bind to the roof and floor of the blister, while EBA antibodies bind solely to the dermal (floor) side of the split skin. ${ }^{1}$

\section{Antigenic Mapping Method}

It is a modified IIF technique using the patient's own skin as a substrate. It is used to determine the site of cleavage or abnormalities in various forms of hereditary epidermolysis bullosa. ${ }^{10}$ The technique is performed with polyclonal/ monoclonal primary antibodies directed against different antigenic components of the dermal-epidermal junction, including the bullous pemphigoid antigen, laminin and type 4 collagen. The cleavage plane is determined by noting which antigens are detected on the roof and the floor of the mechanically induced blisters. All three antigens are seen on the floor (intraepidermal split) in superficial blisters, bullous pemphigoid and laminin are seen on the roof and type 4 collagen on the floor (intralamina lucida split) in junctional blisters, and all three antigens are detected on the roof (sublamina densa split) in dystrophic blisters. ${ }^{1}$

\section{Double Staining Method}

In this method, FITC is used in conjunction with tetramethyl rhodamine isothiocyanate (TRITC) to demonstrate the codistribution of two antigenic substances in tissues. Double staining can be used as a direct/indirect method. The indirect method has very high sensitivity. ${ }^{1}$

\section{Sandwich Technique (Weller, Coons 1954)}

It is a sensitive technique used for the demonstration of the site of the original antibody. Antibody in tissues or smears is reacted with unlabeled antigen. After washing, fluorescent labeled antibody is applied. ${ }^{3}$

\section{Calcium Enhancement Indirect Technique}

The sensitivity of IIF in pemphigus vulgaris and foliaceus may be increased by tris-acetate buffered saline (TAS) with added calcium chloride. In this technique, TAS with $5 \mathrm{mM} \mathrm{CaCl}_{2}$ is used to dilute patients' sera. The mechanism of this heightened sensitivity is unknown. It may occur through stabilization of calcium sensitive epitopes in the target antigens or associated proteins; facilitation of antibody binding or protecting antigens from proteolysis. This technique will not enhance detection of
anti-BMZ antibodies in bullous pemphigoid or epidermolysis bullosa acquisita. $^{2}$

\section{Advantages of Immunofluorescence Technique}

- Simple and reproducible technique

- Short procedure time of 1 to 3 hours

- High sensitivity

- IIF, besides the diagnostic importance, has a substantial prognostic value, particularly for pemphigus cases. ${ }^{5,18}$

\section{Limitations of Immunofluorescence Techniques}

Photobleaching: It is the photochemical destruction of a fluorophore due to the generation of reactive oxygen species in the specimen as a byproduct of fluorescence excitation. ${ }^{6}$

Autofluorescence: Several materials in the dermis, e.g. elastic fibers emit natural autofluorescence, when irradiated by ultraviolet light. ${ }^{5}$ Fixation with aldehydes, particularly glutaraldehyde, can result in high levels of autofluorescence. This can be minimized in fixed cells by washing with $0.1 \%$ sodium borohydride in PBS prior to antibody incubation. ${ }^{6}$ Some of the autofluorescence can be blocked with specific absorptive filters. $^{5}$

Fluorescence overlap: Emission signals overlap occurs when measuring fluorescence of more than one color. These must be electronically removed or the measurement for each detector will overestimate the actual signal. ${ }^{6}$

Nonspecific fluorescence: The pale-green fluorescent background of normal epidermis and dermis reflects the nonspecific binding of the fluorescent antiserum to the tissue. Mild background fluorescence facilitates identification of different histologic structures, which aid in proper localization of the immunoreactants. Background fluorescence can be minimized by using an appropriate dilution of the fluoresceinlabeled antiserum. ${ }^{5}$

\section{A Note on Fluorescence Microscopy}

Immunofluorescent preparations are interpreted using fluorescence microscope immediately or following storage at $4^{\circ} \mathrm{C}$.

Incident light fluorescence microscopy: Modern fluorescence microscopes work on the principle of epifluorescence (illumination of the specimen from above the microscope stage). ${ }^{9}$ The advantages of incidence light microscopy are that the path of the excitation light is through fewer glass surfaces with less light loss due to internal absorption and since the lens used also acts as a condenser, the area viewed equals the area illuminated. ${ }^{4}$

\section{Photography}

Immunofluroscent stain preparations fade quickly, therefore, photodocumentation of freshly stained results is necessary. 
Factors which contribute toward ideal photographs are as follows:

- Unequivocal staining against a good contrast background

- A darkened work area

- Microscope and camera firmly mounted and vibration free

- Usage of mounting media with antifade reagent as they shorten the exposure time significantly

- Preferring camera system that allows $100 \%$ available light to be used and those using short light paths. ${ }^{4}$

\section{CONCLUSION}

Immunofluorescence is the visualization of antigens within cells using antibodies as fluorescent probes. Immunofluorescence combines the specificity of serology and localization of histology. It is an invaluable tool in the diagnosis of immunobullous lesions. The method has achieved the status of combining high sensitivity with high resolution in the visualization of antigens and will be a major tool for many years to come.

\section{ACKNOWLEDGMENT}

We personally thank Dr S Kumar, Dean and Principal of MS Ramaiah Medical College and Hospital, for permitting us to use the facilities available at MS Ramaiah Medical College in preparation of this manuscript.

\section{REFERENCES}

1. Mohan KH, Pai Sathish, Rao Raghavendra, Sripathi H, Prabhu Smitha. Techniques of immunofluorescence and their significance. Indian J of Dermatol, Venereol and Leprol 2008;74(4):415-19.

2. Huilgol SC, Bhogal BS, Black MM. Immunofluorescence of the immunobullous disorders part one: Methodology. Indian J of Dermatol, Venerol and Leprol 1995;61(4):187-95.

3. Cullings CFA. Cullings cellular pathology technique. (4th ed), Butterworths 1984.

4. Bancroft John D. Bancroft theory and practice of histopathological techniques (5th ed), Churchill Livingstone 2002.
5. Zahida Rani, Ijaz Hussain. Immunofluorescence in immunobullous diseases. Journal of Pakistan Association of Dermatologists 2003;13:76-88.

6. Paul Robinson J, Jennifer Sturgis BS, George L, Kumar IHC. Staining Methods (5th Ed), Chapter 10, Immunofluorescence pg 62-65.

7. Rao R, Shenoi SD. Indirect immunofluorescence to demonstrate lichen planus specific antigen (LPSA) in lichen planus. Indian J Dermatol Venerol Leprol 2006;72:350-52.

8. Marx Robert E, Stern Diane. Oral and maxillofacial pathology. Quintessence Publishing Co, Inc 2003;6.

9. Jordan Richard CK, Daniels Troy E, Greenspan John S, Regezi Joseph A. Advanced diagnostic methods in oral and maxillofacial pathology (Part II): Immunohistochemistry and immunofluorescent methods. Oral Surg Oral Med Oral Pathol Oral Radio Endo 2002;93:56-74.

10. Elder David E. Lever's histopathology of skin (9th ed), Lippincott Williams and Wilkins Publications, p 64.

11. Sano Susana Mariela, Quarracino María Cecilia, Aguas Silvia Cristina, González Ernestina Jesús, Harada Laura, Krupitzki Hugo, Mordoh Ana. Sensitivity of direct immunofluorescence in oral diseases. Study of 125 cases. Med Oral Patol Oral Cir Bucal 1 May 2008;13(5):E287-91.

12. Weigand DA. Effects of anatomic region on immunofluorescence diagnosis of bullous pemphigoid. J Am Acad Dermatol 1985;12:274.

13. Srinivas CR, Alfred Sandra. Immunofluorescence in dermatology. Ind J Dermatol 1996;41(2):45-48.

14. Firth Norman A, Rich Alison M, Radden Bryan G, Reade Peter C. Direct immunofluorescence of oral mucosal biopsies: A comparison of fresh-frozen tissue and formalin-fixed, paraffinembedded tissue. Journal of Oral Pathology and Medicine Sep 1992;21(8):358-63.

15. Walker Minz Ranjana, Chhabra Seema, Singh S, Radotra BD, Kumar Bhushan. Direct immunofluorescence of skin biopsy: Perspective of an immunopathologist. Indian J Dermatol Venereol Leprol 2010;76(2):150-57.

16. Kirtsching G, Wojnarowska F. Autoimmune blistering diseases: An update of diagnostic methods and investigations. Clin Exp Dermatol 1994;19:97-112.

17. Vassileva S. Immunofluorescence in dermatology. Int J Dermatol 1993;32:153-61.

18. Ratnam KV, Rang BK. Pemphigus in remission: Value of negative direct immunofluorescence in management. J Am Acad Dermatol 1994;30:547-50. 